\title{
Capillary Electrophoresis in the Analysis of Polyunsaturated Fatty Acids
}

\author{
Hancu Gabriel${ }^{1}$, Tero-Vescan Amelia2*, Filip Cristina², Rusu Aura ${ }^{1}$ \\ Department of Pharmaceutical Chemistry, University of Medicine and Pharmacy Tîrgu Mures, Romania \\ 2 Department of Pharmaceutical Biochemistry, University of Medicine and Pharmacy Tîrgu Mures, Romania
}

The aim of this study to inventory the main electrophoretic methods for identification and quantitative determination of fatty acids from different biological matrices. Critical analysis of electrophoretic methods reported in the literature show that the determination of polyunsaturated fatty acids can be made by: capillary zone electrophoresis, micellar electrokinetic chromatography and microemulsion electrokinetic chromatography using different detection systems such as ultraviolet diode array detection, laser induced fluorescence or mass - spectrometry. Capillary electrophoresis is a fast, low-cost technique used for polyunsaturated fatty acids analysis although their determination is mostly based on gas chromatography.

Keywords: polyunsaturated fatty acids, capillary electrophoresis, omega 3 fatty acids

Received: 13 August 2015 / Accepted: 03 September 2015

\section{Introduction}

Polyunsaturated fatty acids (PUFA) and especially the omega 3 fatty acids (FA) - eicosapentaenoic acid and docosahexaenoic acid and omega 6 FA - arachidonic acid present a special importance in physiological processes; fluidity of biological membranes is influenced by incorporating them into phospholipids structure.

Due to the importance of FAs in different biochemistry and chemistry processes, elaboration of new methods of analysis for their determination represents a challenge and also a necessity for the analysts.

The classical separation technique for FA analysis is the gas chromatography (GC)using flame ionization (FID) or mass spectrometry (MS) detectors. This FA methodology analysis involves firstly lipid fraction extraction, saponification reaction and then derivatization of the total FA content into fatty acid methyl esters (FAME) before injection in the GC equipment. Modern GC methods with high-quality capillary columns allow sensitive and reproducible FA analyses, as well as the characterization of complex mixtures of geometric isomers when combined with other chromatographic separations and spectroscopic identification. However GC analysis of FAshas its drawbacks related to the relatively long analysis times, the use of specialized expensive columns and to the necessity of precoloumn derivatization in order to improve volatility of the samples $[1,2]$.

Although GC is the predominant technique used for FAs analysis, high-performance liquid chromatography (HPLC) has also gained an important role in applications such as the handling of less usual samples, avoidance of degradation of heat-sensitive functional groups, and for micro-preparative purposes [3].

* Correspondence to: Amelia Tero-Vescan

E-mail: amelia.tero.vescan@umftgm.ro
Capillary electrophoresis (CE) is a high-resolution technique used for the separation of complex biological and chemical mixtures; which has gained momentum and emerged as a powerful tool in the analysis of FA in the last decade. In the analysis of PUFA, CE can be considered a viable alternative and also a complementary method to the well-established chromatographic techniques such as HPLC and GC. Although CE was used traditionally for the determination of high-molecular-mass biomolecules, its significance in the analysis of low-molecular-mass ionic species increased rapidly in the last years $[4,5]$.

The aim of the paper is to present a comprehensive review taking into account literature reports regarding analysis of FAs by capillary electrophoresis (CE).

\section{Capillary electrophoresis in the analysis of polyunsaturated fatty acids}

Several CE techniques can be used in the analysis of fatty acids such as capillary zone electrophoresis (CZE), micellar electrokinetic chromatography (MEKC) and microemulsion electrokinetic chromatography (MEEKC) employing different detection systems such as ultraviolet diode array detection (UV-DAD), laser induced fluorescence (LIF) or mass - spectrometry (MS) $[4,5]$.

In order to develop an efficient CE method for the determination of FA, several aspects regarding their ionization have to be taken into consideration. Ionization of the analytes will depend on the composition and especially on the $\mathrm{pH}$ of the background elecrolyte (BGE); consequently the $\mathrm{pH}$ must be generally higher than 7.0 ( $\mathrm{pKa}$ values of fatty acids are around 5.0) in order to promote the dissociation of carboxyl groups and the determination of the analytes in anionic form. An important step in the analysis is the optimization of electrophoretic and analytical parameters: $\mathrm{BGE}$ concentration and $\mathrm{pH}$, type and concentration 
of buffer additives (surfactants, organic solvents, cyclodextrins), applied voltage, system temperature and injection parameters; in order to determine the best conditions for the analysis [6].

Factorial design can be applied as an elegant mathematical tool to optimize the separation of fatty acids by CE, making possible to establish the role of each variable in the determination of optimum conditions and to determine whether variables interact to promote optimal results [6].

A major difficulty of analyzing fatty acids with $\mathrm{CE}$ is their limited solubility in water. As thelength of the alkyl chains increases, the solubility in water decreases, which could result in unstable current or current drops andin capillary clogging during CE separation. Hence, the addition of organic solvents is commonly employed to avoid fattyacid precipitation. Another strategy to improve fatty acid dissolution is by adding urea in the buffer [7].

\section{CZE in fatty acid determination}

CZE is the simplest form of CE, where the separation mechanism is based on differences in the charge-to-mass ratio of the analytes.

The use of CZE for the determination of FAs is limited due to the poor aqueous solubility and low UV absorbance of the analytes. Aqueous electrolytes have been used to resolve separation ofFAs with $\mathrm{C}_{2}-\mathrm{C}_{14}$ by $\mathrm{CZE}$, however for $\mathrm{FA}$ with longer carbon chain the use of MEKC is more adequate.

CE with direct UV detection is problematic and results usually in limited sensitivity, therefore CE with indirect UV detection using an appropria techromophore additive is preferred. CE using direct UV detection is possible if the analytes present one or more double bonds in a linear carbon chain: the presence of a double bonds resulting in a molar absorptivity that is sufficient to achieve an adequate signal peak for detection within the interval from 200 $250 \mathrm{~nm}$.

Saturated FA present low molar absorptivity in the UV, and thus they are analyzed by CZE under indirect UV detection, which is based on the addition of a chromophore agent into the BGE.

Usually the first choice method in the determination of fatty acids seems to be the use of a non-aqueous capillary electrophoresis (NACE) system with indirect UV detection. Some BGE systems for FA determination use also $\mathrm{CD}$ derivatives as buffer additives in order to improve resolution of analytes.

Haddadian et al. [8] developed a CZE method in nonaqueous medium for the separation of various mixtures of saturated and unsaturated free FAs using adenosine monophosphate (AMP) as an indirect photometric detection (IPD) reagent. The advantage of using AMP as an IPD reagent is that AMP has a high molar absortivity, large ratio of background absorbance to background noise; is soluble in aqueous, partially aqueous, and nonaqueous media; therefore, can be used for the separation of different saturated and unsaturated FAs. The separation of FA with $\mathrm{C}_{12}-\mathrm{C}_{31}$ was achieved by using a BGE containing $\mathrm{N}$-methylformamide - dioxane (3:2), $40 \mathrm{mM}$ TRIS, $2.5 \mathrm{mM}$ AMP and $0.5 \%(\mathrm{w} / \mathrm{v})$ Brij in approximately 40 minutes. However, baseline resolution of FA unsaturated isomers $\mathrm{C}_{14}-\mathrm{C}_{22}$ requires the presence of water, because water enhances the solubility, resulting in improved resolution. A mixture of 13 saturated and unsaturated isomers of $\mathrm{C}_{14}-\mathrm{C}_{22}$ FAswas baseline separated in 37 minutes using a mixture of $\mathrm{N}$-methylformamide-dioxane-water (5:4:1).

Porto et al. [9] developed a CZE with direct UV detection at $200 \mathrm{~nm}$ for the determination of omega $3 \mathrm{FA}$ in eggs. The method was used for the qualitative differentiation between natural and enriched chicken eggs through omega 3 FA profiles. The BGE consisted of $12 \mathrm{mM}$ sodium tetraborate mixed with $12 . \mathrm{mM}$ Brij $35,17 \%$ acetonitrile and $33 \%$ methanol, at a $\mathrm{pH}$ of 9.20 . Omega 3FA profiles in chicken egg samples were analyzed by CZE and confirmed by single-quadrupoleMS with an electrospray ionization probe set to negative ionization mode. The results showed that omega 3 FA profiles analyzed by the CZE approach can be used to chemical markers to monitor fraud, presenting simplicity, short analysis time $(10 \mathrm{~min})$ and low cost as advantages.

Bannore et al. [10] showed the suitability of CZE for the analysis of free FAs in peanuts seeds. A partially aqueous BGE consisting of $40 \mathrm{mMTris}, 2.5 \mathrm{mM}$ AMP and $7 \mathrm{mM} \alpha-\mathrm{CD}$ in ( $\mathrm{N}$-methylformamide) NMF/ dioxane/ water $(5: 3: 2 \mathrm{~V} / \mathrm{V})$ mixture at a $\mathrm{pH} 9.0$ was used for the separation. While AMP served as the background UV absorber for indirect UV detection of the analytes, $\alpha-C D$ functioned as the selectivity modulator by affecting the relative effective electrophoretic mobilities of the various FAs due to their differential affinities towards $\alpha-C D$. The CZE method allowed the screening of peanut seeds for their content in oleic and linoleic acids, which is essential in breeding of peanuts with high-oleic acid content. The extraction method of FAs from peanut seeds proved to be very reproducible with a high recovery approaching quantitative yield ( $97 \%$ recovery).

An alternative method for extraction optimization of C18:2 n-6 and C18:3 n-3, the main precursors for the synthesis of conjugated linoleic acid, in Brachiaria ruzizienses forages was proposed by Castro el al [11]. The optimization extraction was monitored by quantification of C18:2 n-6 and C18:3 n-3 through a CZE method. The results obtained by CZE were compared to the GC results in real samples using the paired t-test. No significant difference between methods was found within a $95 \%$ confidence interval ( $\mathrm{p}$-value $=0.937)$. The alternative CZE method for Brachiariar uzizienses forages analysis has some advantages in comparison with official GC method such as, short analysis time (10 $\mathrm{min})$, no derivatization step for sample preparation, absence of specific separation columns, lower analytical cost and high throughput. 


\section{MEKC in fatty acid determination}

The separation principle in MEKC is based on the addition to the BGE solution of a micellar "pseudostationary" phase, which interacts with the analytes according to partitioning mechanisms. The "pseudostationary" phase is composed of a surfactant added to the buffer solution in a concentration above its critical micellar concentration (CMC). The charged "pseudostationary" phase moves through the capillary under an applied voltage at an electrophoretic velocity that is proportional to the charge-tosize ratio; consequently the separation of charged analytes is based on their charge-to-size ratio, while that of neutral analytes is based on their differential partitioning into the micellar phase.

FA that have short alkyl chains are relatively less hydrophobic and are easier to separate, however as the length of the chain increases their hydrophobicity also increases and their solubility in aqueous BGE eventually becomes a limiting factor. In these cases MEKC can be used to separate long-chain highly hydrophobic FA.

This type of separation, featuring anionic analytes and neutral micelles, can be conveniently modeled by adapting the physico-chemical framework for the case of neutral analytes and anionic micelles.

In this context Collet and Gareil [12] studied the separation of saturated and unsaturated long chain $\left(\mathrm{C}_{14}-\mathrm{C}_{20}\right)$ free FAs by MEKC in the presence of Brij 35 micelles and methanol. The detection was performed by indirect absorbance detection using $p$-methoxybenzoate as absorbing co-ion in the electrolyte. It has been established that optimal resolution is obtained when the anionic analytes spend more time in the bulk hydroorganic electrolyte than in the neutral micellar pseudo-phase. This approach was implemented for optimizing the Brij 35 concentration and the methanol content in the electrolyte, and allowed the authors to determine chromatographic-type selectivity, which is a major issue for the assessment of alternate separation methods. Finally, this work demonstrateted that MEKC is a cost and time effective method for the separation of critical pairs of saturated and unsaturated FFAs of identical equivalent chain length.

Soliman et al. [13] developed a simple direct MEKC method for the simultaneous determination of 15 omega- 3 (n-3) and omega 6 (n-6) relevant fatty acids. The separation was achieved using $40 \mathrm{mMsodium}$ borate buffer at $\mathrm{pH} 9.50$ containing $50 \mathrm{mMsodium}$ dodecyl sulphate (SDS), $10 \mathrm{mM}$ $\beta$-cyclodextrin, and $10 \%$ acetonitrile.The presence of two or more double bonds in their structures allows these fatty acids to absorb light at $214 \mathrm{~nm}$, which eliminated possible spectral interference from saturated and monosatuated fatty acids present in the analyzed food samples. The developed CE method has LODs of $<5 \mathrm{mg} / \mathrm{L}$ and good linearity (R> 0.980 ) for all fatty acids studied. The proposed method was successfully applied to the determination of $n-3$ and $n-6$ fatty acids in flax seed, commercially available oils and a selection of grass-fed and grain-fed beef muscle samples.
A cyclodextrin-modified micellar electrokinetic chromatography (CD-MEKC) method was developed by Liu et al. [14] for separating conjugated linoleic acid isomers. All the seven CLA isomers were completely separated in the optimized conditions $80 \mathrm{mM}$ sodium tetraborate, $54 \mathrm{mMS}$ DS, $8 \mathrm{M}$ urea, $4 \%(\mathrm{v} / \mathrm{v})$ ethanol, $4 \%(\mathrm{w} / \mathrm{v}) \beta$-cyclodextrin at $\mathrm{pH}$ 9.0. The CD-MEKC method was superior to the gas chromatographic (GC) and silver-ion high-performance liquid chromatographic $(\mathrm{Ag}(+)-\mathrm{HPLC})$ methods that were generally used in analyzing linoleic acid isomers.

An alternative methodology for simultaneous analysis of majority cis-trans fatty acids such as stearic, oleic, palmitic, linoleic and linolenic by CZE under indirect detection was proposed by Barra et al. [15]. The CZE method was optimized through the $2^{3}$ central composite design $\left(2^{3} \mathrm{CCD}\right)$ with three replicates in central point, having as optimization factors Brij 35, acetonitrile and 1-octanol. The BGE for the optimum separation condition consisted of: 15 $\mathrm{mM} \mathrm{NaH} \mathrm{PO}_{4} / \mathrm{Na}_{2} \mathrm{HPO}_{4}$ buffer at $\mathrm{pH}$ 6.86, 4 mMsodium dodecyl benzene sulphoneate (SDBS), 8.3 mMBrij 35 and $45 \% \mathrm{v} / \mathrm{v}$ of acetonitrile, and $2.1 \%$ of 1 -octanol. The FA quantification was performed through response factor $\left(\mathrm{R}_{\mathrm{f}}\right)$ approach, which provided high analytical throughput for the real samples analysis. The CE method optimized was successfully applied to the analysis of FA in samples of olive oil, soy oil, hydrogenated vegetable fat, butter, margarine and filled cookie. The results obtained were compared with the GC official method through paired sample t test and no significant difference was found within $95 \%$ confidence interval.

\section{Conclusions}

CE seems to represent an interesting alternative in the screening analysis and quantitative determination of fatty acids from different matrices, offering advantages related to the high separation efficiency, low consumption of samples and reagents, short analysis time, rapid method development, simple sample preparation without derivatization; all these aspects making this technique very attractive for routine preliminary analysis.

On the other hand CE presents much lower sensibility and still a limited range in the analysis of fatty acids in comparison with the chromatographic techniques (HPLC or GC).Also due to low solubility of fatty acids in aqueous buffer, high amounts of organic solvent in the separation medium are often required; which can decrease micelle size, and in certain circumstances, even depress micelle formation. Also in HPLC, mobile phase gradients are easily established and the collection of fractions for further examination is possible unlike in $\mathrm{CE}$ where the analyte loadability is small.

It is evident that $\mathrm{CE}$ have great potential in the determination of FA; the difficulty remains in identifying optimized buffer systems which can resolve an entire crosssection of FA and also in the identification of sensitive detection systems; which curtailed the widespread use 
of $\mathrm{CE}$ in quality control of foods, pharmaceuticals, and chemical industry as well as extremely sensitive diagnostic protocols in medicine.

\section{Acknowledgment}

This paper was published under the frame of European Social Found, Human Resources Development Operational Program 2007-2013, project no. POSDRU/159/1.5/S/136893

\section{References}

1. Seppänen-LaaksoT, Laakso I, HiltunenR. Analysis of fatty acids by gas chromatography, and its relevance to research on health and nutrition. Anal.Chim.Acta. 2002;465,39-62.

2. Bailey-Hall E, Nelson EB, Ryan AS.Validation of a rapid measure of blood PUFA levels in humans. Lipids 2008;43,181-186.

3. Brondz I. Development of fatty acids analysis by high-performance liquid chromatography, gas chromatography and related techniques. Anal. Chim. Acta. 2002;465,1-37.

4. Otieno AC,Mwongela SM. Capillary electrophoresis-based methods for the determination of lipids - A review. Anal. Chim. Acta. 2008;624,163174.

5. de Oliveira MAL, Porto BLS,Faria IDL et al. 20 years of fatty acid analysis by capillary electrophoresis. Molecules 2014;19,14094-14113.

6. de Oliveira MAL, Micke GA, Bruns R, Tavares MFM. Factorial design of electrolyte systems for the separation of fatty acids by capillary electrophoresis. J. Chromatogr. A 2001;924,533-539.
7. Tavares M,Jager A, Silva C et al.Applications of capillary electrophoresis to the analysis of compounds of clinical, forensic, cosmetological, environmental, nutritional and pharmaceutical importance. J. Braz. Chem. Soc. 2003;14,281-290.

8. Haddadian F,Shamshi SA, Warner IM. Separation of saturated and unsaturated free fatty acids using capillary electrophoresis with indirect photometric detection. J. Chrom. Sci. 1999;37,103-107.

9. Porto BLS, de Souza MVN, de Oliveira MAL. Analysis of Omega 3 fatty acid in natural and enriched chicken eggs by capillary zone electrophoresis.Anal. Sci. 2011;27,541-546.

10. Bannore YC, Chenault KD, Melouk HA, Rassi Z. Capillary electrophoresis of some free fatty acids using partially aqueous electrolyte systems and indirect UV detection. Application to the analysis of oleic and linoleic acids in peanut breeding lines. J. Sep. Sci. 2008;31,2667-2676.

11. Castro RDC, Sobrinho FS, da Gama MAS, et al. Analysis of the main conjugated linoleic acid (CLA) precursors (C18:2 n-6 and C18:3 n-3) in Brachiaria ruzizienses by capillary zone electrophoresis. Cent. Eur. J. Chem. 2013;11,1286-1296.

12. Collet J, Gareil P. Micellar electrokinetic chromatography of long chain saturated and unsaturated free fatty acids with neutral micelles Considerations regarding selectivity and resolution optimization. J. Chromatogr. A 1997;792,165-177.

13. Soliman LC, Donkor KK, Church JS et al. Separation ofdietary omega-3 and omega- 6 fatty acids in food by capillary electrophoresis. J. Sep. Sci. 2013;36,3440-3448.

14. Liu XH, Cao YS, Chen Y. Separation of conjugated linoleic acid isomers by cyclodextrin-modified micellar electrokinetic chromatography. J. Chromatogr. A 2005;1095,197-200.

15. Barra PMD, Castro RDC, de Oliveira PL et al. An alternative method for rapid quantitative analysis of majority cis-trans fatty acids by CZE. Food Res. Int. 2013;52,33-41. 